\title{
A Hybrid Approach for Large-scale Fabrication of Paper-based Electrochemical Assays for Biomedical Diagnosis
}

\author{
Fariba Ghaderinezhad ${ }^{1}$, Savas Tasoglu $1,2,3,4,5^{*}$ \\ ${ }^{1}$ Department of Mechanical Engineering, ${ }^{2}$ Department of Biomedical Engineering, ${ }^{3}$ Institute of Materials Science, \\ ${ }^{4}$ Institute for Collaboration on Health, Intervention, and Policy, ${ }^{5}$ The Connecticut Institute for the Brain and \\ Cognitive Sciences, University of Connecticut, 337 Mansfield Rd, Storrs, CT 06269. \\ *savas.tasoglu@uconn.edu
}

Received: 20 March 2019

Accepted: 18 September 2019

DOI: $10.18466 /$ cbayarfbe. 542120

\begin{abstract}
The electrochemical method offers a sensitive, portable, and cost-effective method for detecting a range of analytes and has the capacity to be miniaturized. However, according to the World Health Organization, there is a need for point-of-care devices that meet the criteria for medical diagnostics in remote and resourcelimited regions, as set forth by the World Health Organization. In this context, we developed a low-cost and high-throughput method for fabricating paper-based devices for electrochemical assays using a desktop pen plotter. In this study, we used a permanent marker to plot hydrophobic barriers and a liquid dispenser to pattern three electrodes (working, counter, and reference electrodes) with carbon paste on a paper substrate. To test the performance of the fabricated electrochemical devices, chronoamperometric and cyclic voltammetric analyses of potassium ferricyanide was conducted. Further, to demonstrate of the possible biomedical diagnostic applications, a quantitative glucose assay was performed. Here, we presented a lowcost electrochemical device fabrication method convenient for large-scale fabrication and widespread implementation of paper-based technologies in developing countries and resource-limited settings.
\end{abstract}

Keywords: Pen plotter, paper-based assays, electrochemical, glucose assay, high-throughput, biosensor.

\section{Introduction}

In the development and evaluation of point-of-care devices, the World Health Organization (WHO) established a set of criteria that are collectively referred to as ASSURED: Affordable, Sensitive, Specific, Userfriendly, Rapid and robust, Equipment-free, and Deliverable to end-users [1]. Existing medical diagnostic technologies have limitations to fulfill the needs of remote and resource-limited regions due to their complicated clinical infrastructure, long turnaround time, and relatively high costs; thus, new medical diagnostic technologies must be developed [2]. Paper-based assays are promising tools in that they are widely accessible, can be easily produced at high-throughput, and are disposable and cost-effective [2-7]. Colorimetric detection is one of the most widely used detection methods. Recent studies have presented novel methods to address the inhomogeneity of color [8] and to obtain quantitative measurements [9]. However, the electrochemical detection (ECD) method has also attracted much attention as it is sensitive, inexpensive, portable, and can be miniaturized. Unlike the colorimetric method, ECD can function with impure (i.e., glucose in urine) samples and is not sensitive to changes in the ambient illumination $[2,10]$. Moreover, ECD requires only simple instrumentation and a small amount of electrical power, making it compatible with use in the field [11]. However, the remaining hurdle to the widespread implementation of paper-based technologies in developing countries is the development of a simple, high-throughput, and lowcost fabrication method [10].

Paper-based devices for electrochemical assays are composed of electrodes and a paper substrate [11,12]. Among the methods developed for plotting electrodes on paper are sputtering [13], sintering [14], inkjet printing $[15,16]$, screen printing $[11,17]$, and direct plotting with a conductive pen or pencil $[10,18,19]$. For example, Ghosale et al. proposed a method for writing electrodes using a ball-point pen filled with silver nanoparticles capped with octylamine [20]. In another study, Ruecha et al. used an inkjet printer to fabricate entire paper-based potentiometric devices including the electrodes [16].

Here, we propose a low-cost, high-throughput, singlestep fabrication method based on a desktop pen plotter integrated with a liquid dispenser [3]. We used permanent markers and carbon paste to plot the hydrophobic barriers and the electrodes, respectively. A syringe was filled with carbon paste and was connected to a liquid dispenser and the paste flow was driven by the application of a fixed air pressure (Fig 1). The paper was hot-laminated on one side to improve its durability, 
stability, and mechanical strength and provide stability to the hydrophobic barriers and bio-chemical reagents during storage [3]. The electrochemical operation of the fabricated paper-based assays was validated by both cyclic voltammetry (CV) and chronoamperometry (CA) with potassium ferricyanide. These electrochemical techniques measure the current in an electrochemical cell that develops when a potential is applied. In $\mathrm{CV}$, the voltage potential changes linearly from defined start voltage to the end voltage once or in consecutive cycles. In CA, a step voltage is applied at the beginning of the experiment and kept constant during measurement. Additionally, CA analysis of glucose was conducted to demonstrate a possible diagnostic application of the fabricated paper-based devices for electrochemical assays.

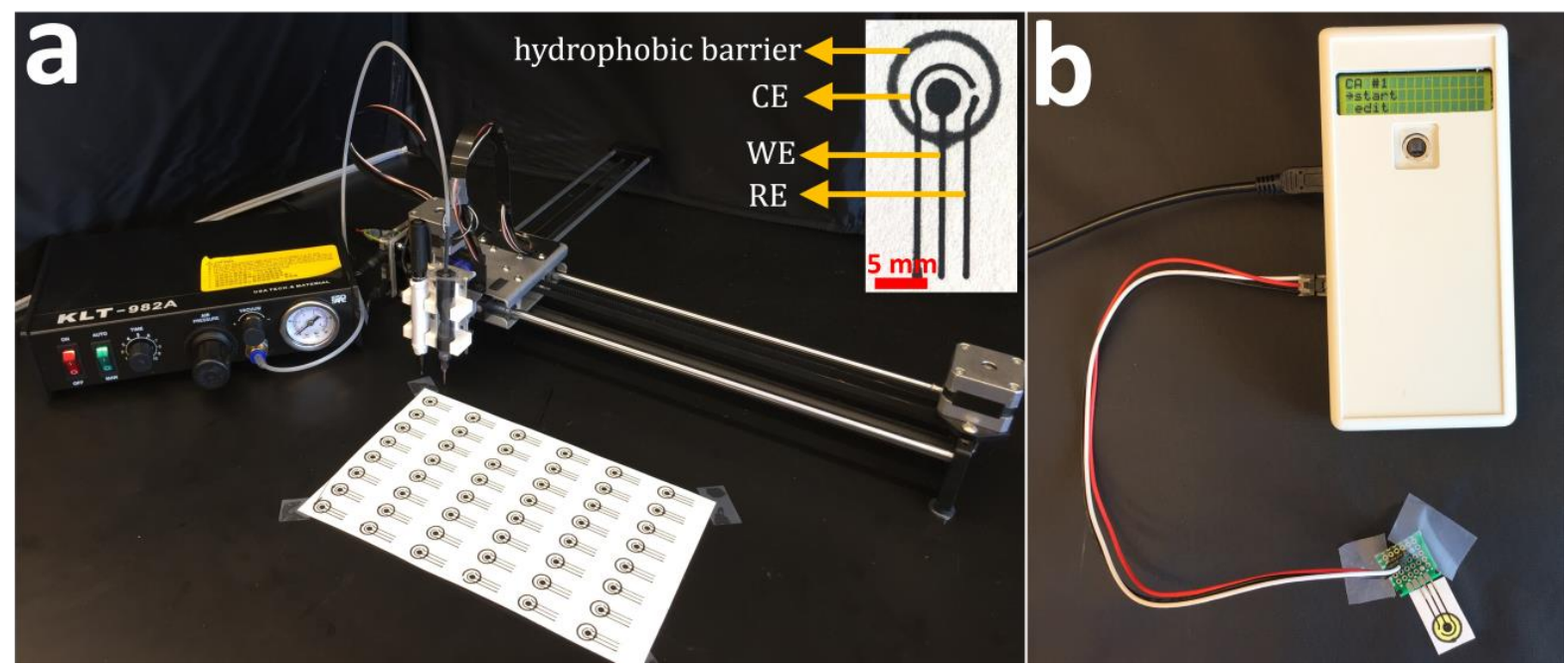

Figure 1. Fabrication setup for the paper-based electrochemical devices. a) Desktop pen plotter integrated with a syringe holder. Also depicted is the liquid dispenser controller used to drive the flow of the carbon ink from the syringe. The inset shows the fabricated paper-based devices for electrochemical assays with three carbon electrodes (working: WE, counter: $\mathrm{CE}$, and reference: RE) and a hydrophobic barrier patterned by a Comix marker. b) Handheld potentiostat used for cyclic voltammetry (CV) and chronoamperometry (CA) analyses. The potentiostat is connected to one of the fabricated paper-based devices.

\section{Materials and Methods}

\subsection{Materials}

Desktop pen plotter (AxiDraw) from Evil Mad Scientist Laboratories, CA, US; Double-tipped marker (Comix) from Comix Group Co. Ltd., Shenzhen, China (the fineand broad-tips are 0.5 and $2.0 \mathrm{~mm}$ in diameter, respectively); Chromatography paper (Whatman No. 1) from GE Healthcare Life Sciences, IL, US; Writing paper (Clairefontaine Thriomphe and Rhodia) from Clairefontaine-Rhodia, France; Hot-laminating layer (GBC EZUse thermal laminating pouches) from Swingline, IL, US; Benchtop laminator (GBC Inspire) from Swingline, IL, US; Potentiostat (Cheapstat) from IO Rodeo Inc, CA, USA; Liquid Dispenser from Advance Electrical Equipment Co. Ltd., Taiwan; Potassium ferricyanide (III) $\left(\mathrm{K}_{3}\left[\mathrm{Fe}(\mathrm{CN})_{6}\right], \quad 702587\right)$, glucose oxidase (G7141), and potassium chloride (746436) from Sigma-Aldrich, MO, US; Pure glucose from Modernist Pantry LLC., NH, US; Phosphate Buffered Saline (PBS, pH 7.4) from Fisher Scientific, MA, US; Carbon paste from Bare Conductive, London, UK; Screen-Printed Carbon Electrode (DRP 110) from Metrohm AG, Herisau, China.

\subsection{Device Fabrication}

To enable a simple, one-step fabrication, a liquiddispensing plotter was created by integrating a liquid dispenser with an AxiDraw pen plotter. The driver software of the AxiDraw plotter was modified to add another output that can be used to control a liquid dispenser in synchrony with the motion of the pen. The amount of carbon paste deposited was controlled by adjusting the air pressure of the liquid-dispensing controller and the speed of the plotter and, once the desired flow rate is achieved, the pressure was held constant and the dispenser was moved along a 2D path at a constant speed by AxiDraw $(10 \mathrm{~mm} / \mathrm{s})$. Three different papers were used as the substrate: a porous Whatman chromatography paper and two different coated writing papers, Clairefontaine and Rhodia. The pattern for the three electrodes (working, counter, and reference) was designed in Inkscape (Free Software Foundation, Inc., MA, US) and plotted with the carbon paste. Then, using the fine tip of a Comix marker, a $10 \mathrm{~mm}$-diameter circle was plotted on paper as the hydrophobic barrier of the detection zone. After plotting the electrodes, the paper was placed on a $90^{\circ} \mathrm{C}$ hotplate overnight to set the carbon 
paste. Then, a laminating layer was applied to the side opposite the plotted patterns by hot lamination.

\subsection{Electrochemical measurements}

The electrochemical operation of the fabricated devices was validated using potassium ferricyanide (III) $\left(\mathrm{K}_{3}\left[\mathrm{Fe}(\mathrm{CN})_{6}\right]\right)$ and glucose. The electrochemical measurements (CV and CA analyses) were carried out using a potentiostat at the room temperature. $\mathrm{CA}$ and $\mathrm{CV}$ analyses of $\mathrm{K}_{3}\left[\mathrm{Fe}(\mathrm{CN})_{6}\right]$, were carried out at different scan rates (for $\mathrm{CV}$ ) and step voltages (for $\mathrm{CA}$ ). The $\mathrm{K}_{3}\left[\mathrm{Fe}(\mathrm{CN})_{6}\right]$ samples were prepared by dissolving various concentrations of $\mathrm{K}_{3}\left[\mathrm{Fe}(\mathrm{CN})_{6}\right]$ in a 1 M potassium chloride $(\mathrm{KCl})$ solution (the supporting electrolyte). CA analyses were performed on devices on which $50 \mu \mathrm{l}$ of the $K_{3}\left[\mathrm{Fe}(\mathrm{CN})_{6}\right]$, sample was deposited in the detection zone and on those which were submerged in the same sample. For the glucose tests, the devices were prepared by depositing $10 \mu \mathrm{l}$ of a solution of 300 $\mathrm{U} / \mathrm{ml}$ glucose oxidase and $20 \mathrm{mM} \mathrm{K}_{3}\left[\mathrm{Fe}(\mathrm{CN})_{6}\right]$ (the mediator) in PBS in the detection zone and drying at room temperature for $10 \mathrm{~min}$. The $\mathrm{CA}$ analysis was then conducted by depositing $50 \mu \mathrm{l}$ of a glucose sample dissolved in PBS. The mechanism for glucose detection involves glucose oxidation by the catalytic activity of glucose oxidase, leading to the reduction of $\mathrm{Fe}$ (III) to $\mathrm{Fe}$ (IV) and the generation of $\mathrm{Fe}(\mathrm{CN})_{6}^{4-}$ ions, which are detected by the electrochemical analysis (Equations 1-3).

$$
\begin{aligned}
& \text { glucose }+2 \mathrm{~K}_{3}\left[\mathrm{Fe}(\mathrm{CN})_{6}\right]+\mathrm{H}_{2} \mathrm{O} \\
& \stackrel{\text { glucose oxidase }}{\longrightarrow} \text { gluconic acid }+2 \mathrm{~K}_{4}\left[\mathrm{Fe}(\mathrm{CN})_{6}\right] \\
& 2 \mathrm{Fe}(\mathrm{CN})_{6}^{4-} \stackrel{\text { anodic electrode }}{\longrightarrow} 2 \mathrm{Fe}(\mathrm{CN})_{6}^{3-}+2 e^{-} \\
& 2 \mathrm{Fe}(\mathrm{CN})_{6}^{3-}+2 e^{- \text {cathodic electrode }} \longrightarrow \mathrm{Fe}(\mathrm{CN})_{6}^{4-}
\end{aligned}
$$

To compare the performance of the fabricated devices with the performance of commercially available screenprinted electrodes (SPEs), CA analyses were performed with different concentrations of $\mathrm{K}_{3}\left[\mathrm{Fe}(\mathrm{CN})_{6}\right]$ and glucose. Unlike the devices fabricated by the proposed method, the electrodes in the SPEs were screen-printed with silver/silver chloride $(\mathrm{Ag} / \mathrm{AgCl})$ and the portions of the working and counter electrodes in contact with samples were covered by screen-printed carbon. However, we were not able to immobilize the glucose reagents on the ceramic surfaces of the SPEs. Thus, $10 \mu 1$ of the glucose oxidase and $K_{3}\left[\mathrm{Fe}(\mathrm{CN})_{6}\right]$ solution were dried on a $6 \times 8 \mathrm{~mm}$ piece of chromatography paper at room temperature. The paper was placed on the detection zone of the SPE, $50 \mu \mathrm{l}$ of the glucose sample was deposited on this paper, and the CA analyses were carried out by applying a $300 \mathrm{mV}$ potential.

\section{Results and Discussion}

Comparing the different paper substrates, the results were more consistent with the coated papers. We hypothesize that this is because the coated papers absorb the liquid sample more slowly than the relatively porous chromatography paper such that the sample remains above the carbon electrodes rather than surrounding them from all sides, as in the SPEs. The amount of sample wicked by the paper depends on how long the sample is placed before starting the test. With coated papers this process is slower resulting less dependency on testing time. It was also found that the plotted edges were more contained within the plotted lines on the coated papers than on the chromatography paper due to smoother surface of the papers.

To check the zero-voltage response of the fabricated devices, $1 \mathrm{mM}$ of $\mathrm{K}_{3}\left[\mathrm{Fe}(\mathrm{CN})_{6}\right]$ and $1 \mathrm{M} \mathrm{KCl}$ were applied as the sample and dried. At $0 \mathrm{~V}$, there was a negligible current on the devices that are left on a $90^{\circ} \mathrm{C}$ hotplate overnight. However, in the devices that were set using the heat from the laminator $\left(\sim 140^{\circ} \mathrm{C}\right)$, the $0 \mathrm{~V}$ response was nearly $300 \mathrm{mV}$. This is likely because the solvent of the carbon paste did not fully evaporate on the laminator due to short exposure time to heat, and the remaining chemicals caused this response; and these devices were used throughout the project.

Then, the electrochemical behavior of the fabricated paper-based devices was validated by $\mathrm{CV}$ and $\mathrm{CA}$ with a model electroactive compound, $5 \mathrm{mM} \mathrm{K}_{3}\left[\mathrm{Fe}(\mathrm{CN})_{6}\right]$ in 1 $\mathrm{M} \mathrm{KCl}$ at various scan rates between 50 and $500 \mathrm{mV} / \mathrm{s}$. The CV results (Fig 2a) showed that as the scan rate was increased, the peak current (defined as the anodic, $\mathrm{I}_{\mathrm{p}}$, and cathodic, $\mathrm{I}_{-\mathrm{p}}$, peak currents) increased and that relationship is consistent with the Randles-Sevcik equation $\left(\mathrm{I}_{\mathrm{p}}\right.$ is proportional to the square root of the scan rate). The $I_{p} / I_{-p}$ ratio was close to unity for all scan rates tested and the peak shapes matched the characteristic graph of a typical reversible (Nernstian) electrochemical reaction. It should be noted that as the scan rate increased from 50 to $500 \mathrm{mV} / \mathrm{s}$, the difference between the voltages at which the peaks occur increased from 120 to $320 \mathrm{mV}$. However, theoretically, for reversible reactions that transfer one electron, including the redox-active couple of $\mathrm{Fe}(\mathrm{CN})_{6}^{3-} / \mathrm{Fe}(\mathrm{CN})_{6}^{4-}$, this difference should be only $57 \mathrm{mV}$ and should be independent of the scan rate. This observed discrepancy generally occurs when the Nernstian equilibrium cannot be maintained over the course of the reaction, causing the current peaks to occur at voltages that are farther apart as the scan rate is increased. Moreover, the results show that the difference between the voltages at which the peak currents occur depends on the scan rate. This indicates that the electrontransfer kinetics was slow relative to the voltage scan rate. This type of reaction is referred as quasi-reversible or irreversible electron transfer reactions. 

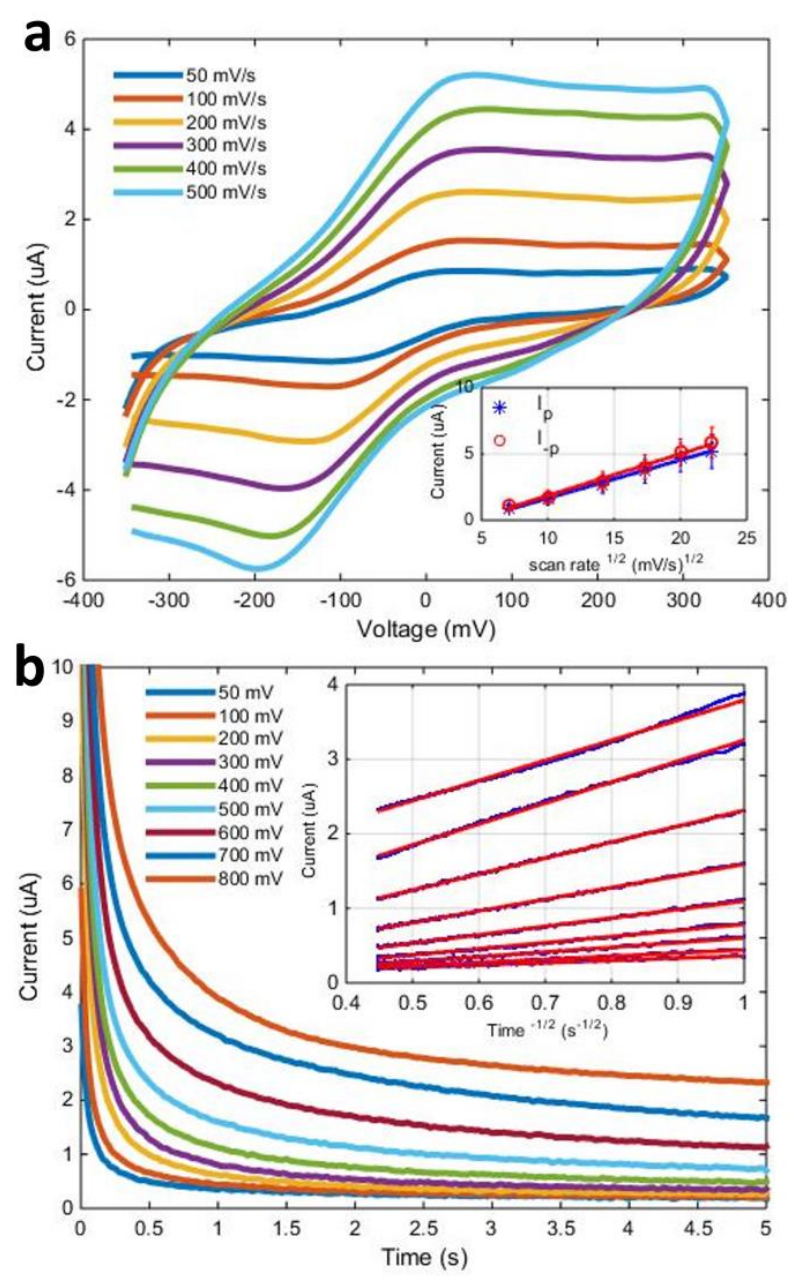

Figure 2. Electrochemical characterization of the paperbased devices for electrochemical assays. a) Cyclic voltammetry results for $5 \mathrm{mM} \mathrm{K}_{3}\left[\mathrm{Fe}(\mathrm{CN})_{6}\right]$ in $1 \mathrm{M} \mathrm{KCl}$ obtained at different scan rates: 50, 100, 200, 300, 400, and $500 \mathrm{mV} / \mathrm{s}$. The inset shows the anodic $\left(\mathrm{I}_{\mathrm{p}}\right)$ and cathodic $\left(\mathrm{I}_{-\mathrm{p}}\right)$ peak currents versus the square root of the scan rate and the solid line represents a linear fit to this data with the equations $\mathrm{y}=0.28938 \mathrm{x}-1.2309\left(\mathrm{R}^{2}=\right.$ $0.99529)$ and $y=0.31432 x-1.279\left(R^{2}=0.99118\right)$, respectively; $(\mathrm{n}=10)$. b) Chronoamperometry result for $5 \mathrm{mM} \mathrm{K} \mathrm{K}_{3}\left[\mathrm{Fe}(\mathrm{CN})_{6}\right]$ in $1 \mathrm{M} \mathrm{KCl}$ at different step voltages: 50, 100, 200, 300, 400, 500, 600, 700, and 800 $\mathrm{mV}$. The inset shows the current versus the negative square root of time with the solid red lines representing linear fits to this data; $(n=10)$.
CA was also tested because it has a higher signal-to-noise ratio compared to $\mathrm{CV}$. CA analysis was performed on the same model electroactive compound (5 $\mathrm{mM}$ $\mathrm{K}_{3}\left[\mathrm{Fe}(\mathrm{CN})_{6}\right]$ in $\left.1 \mathrm{M} \mathrm{KCl}\right)$ at different step voltages between 50 and $800 \mathrm{mV}$. The results (Fig. 2b) show that the current increased as the step voltage was increased. In addition, the current decay was proportional to $\mathrm{t}^{-1 / 2}$, which is consistent with the Cottrell equation ( $I=$ $n F A C \sqrt{D} / \sqrt{\pi t}$ where $\mathrm{I}$ is the current, $\mathrm{n}$ is the number of electrons released from each molecule, $\mathrm{F}$ is the faraday constant, A is the surface area of the working electrode, $\mathrm{C}$ is concentration of the chemical of interest, and D is the diffusion constant. The steady-state (final) current values were found to vary greatly with the resistive current, which is dominantly affected by the solvent concentration. The effect of the diffusion is the only transient current inducing effect, and it is not dominated by any other effect caused by the solvent. Thus, by fitting the Cottrell equation to the transient data, the effect of the solvent was minimized. From the fitted curves of standard samples, a calibration constant, $\mathrm{k}$ (= $n F A C \sqrt{D} / \sqrt{\pi}$ ), was derived, in which only the concentration $(\mathrm{C})$ is variable if the same type of paper and analyte are used. Thus, the sample concentration can be calculated based on the Cottrell fit obtained for an unknown sample based on the value of $\mathrm{k}$ derived from the calibration experiments, which is detailed in the next paragraph.

CA analysis was conducted on different concentrations of $\mathrm{K}_{3}\left[\mathrm{Fe}(\mathrm{CN})_{6}\right]$ using both the fabricated devices and the SPEs. The results obtained by pipetting the sample onto each of the devices showed that the steady-state current increased as the concentration increased (Fig 3a, b). Moreover, these results were compared with the results obtained by dipping the fabricated devices into the same samples (Fig 3c). Comparing the measurements, it was found that depositing the sample resulted in more consistent results as evidenced by a smaller R-squared value for the linear fit to the concentration-versus-current data while dipping into the sample resulted in a higher standard deviation over repeated measurements and lower current readings. Further details regarding LOD of SPEs reported by the manufacturer can be found here: https://www.metrohm.com/en/products/electrochemistry /electrochemistry-electrodes/. 

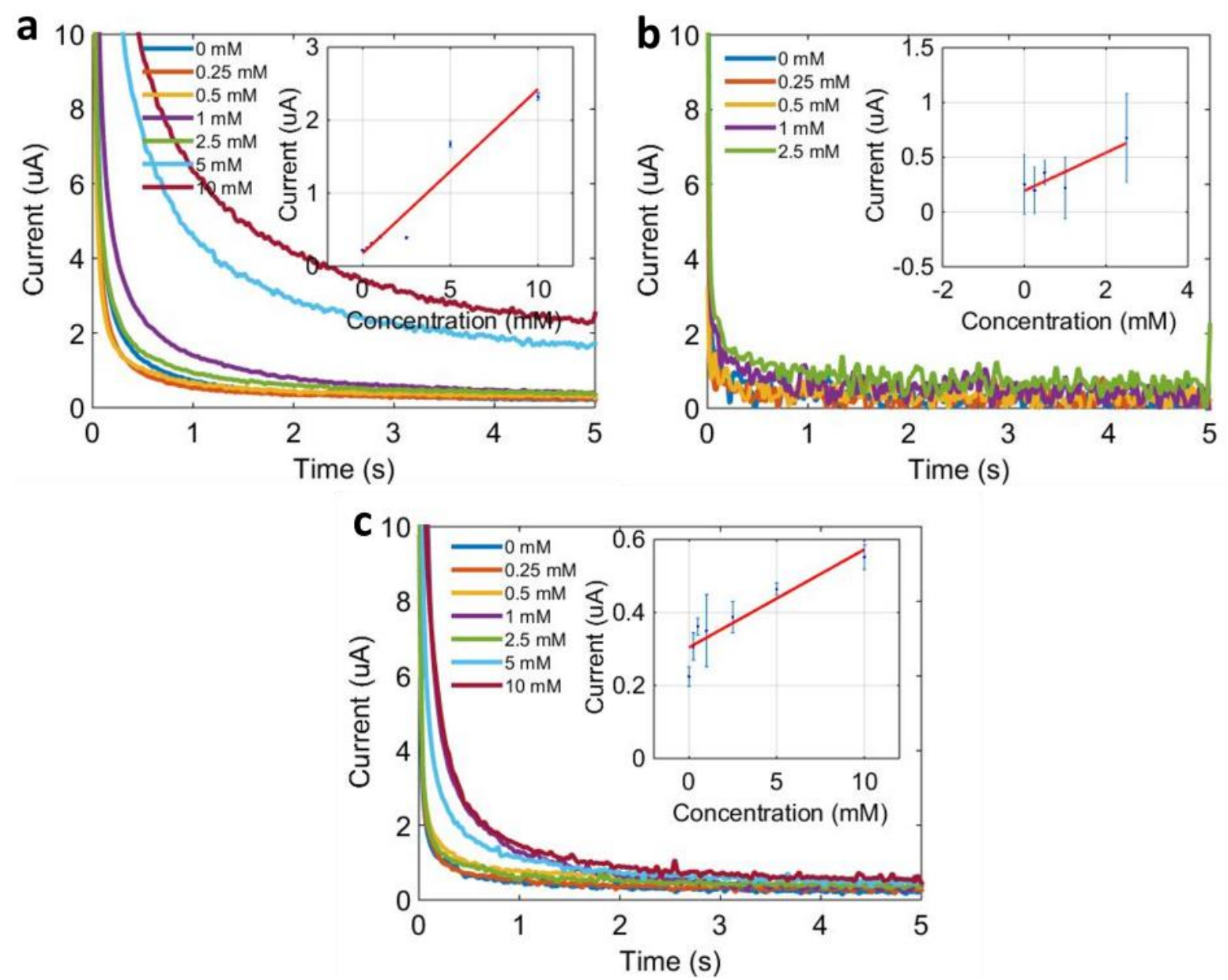

Figure 3. Chronoamperometry results for different concentrations of $K_{3}\left[\mathrm{Fe}(\mathrm{CN})_{6}\right]$ in $1 \mathrm{M} \mathrm{KCl}$ obtained by depositing the sample a) the fabricated paper-based devices and (b) commercial screen-printed electrodes; and c) by dipping the fabricated devices into the sample. The insets show the steady-state currents for the range of concentrations and the solid lines represent linear fits to the data with the following equations: a) $y=0.22484 x+0.17608\left(R^{2}=0.93673\right)$, b) $\left.\mathrm{y}=0.19295 \mathrm{x}+0.1699\left(\mathrm{R}^{2}=0.98655\right), c\right) \mathrm{y}=0.026825 \mathrm{x}+0.30412\left(\mathrm{R}^{2}=0.85059\right),(\mathrm{n}=5)$.

Finally, the performance of the fabricated devices was tested for a common diagnostic application: glucose testing. A glucose assay was conducted using both the paper-based devices and the SPEs. The effect of the reaction time on the CA results was investigated because the chemical/enzymatic reaction responsible for the electron transfer in the glucose assay requires time to reaction to take place. The CA results obtained from the fabricated device with $5 \mathrm{mM}$ glucose (Fig 4a) show that the measured current increased as the reaction time increased. This is because the glucose concentration increases with the reaction time. After fixing the reaction time variable, glucose samples are tested in the range of 0 to $20 \mathrm{mM}$ (Fig 4b, c), which is relevant to the range of glucose concentrations found in urine. The results show the current increased linearly with the glucose concentration in both the fabricated devices and the SPEs although the sensitivity of the SPEs was higher than that of the fabricated devices (Fig 4c). 

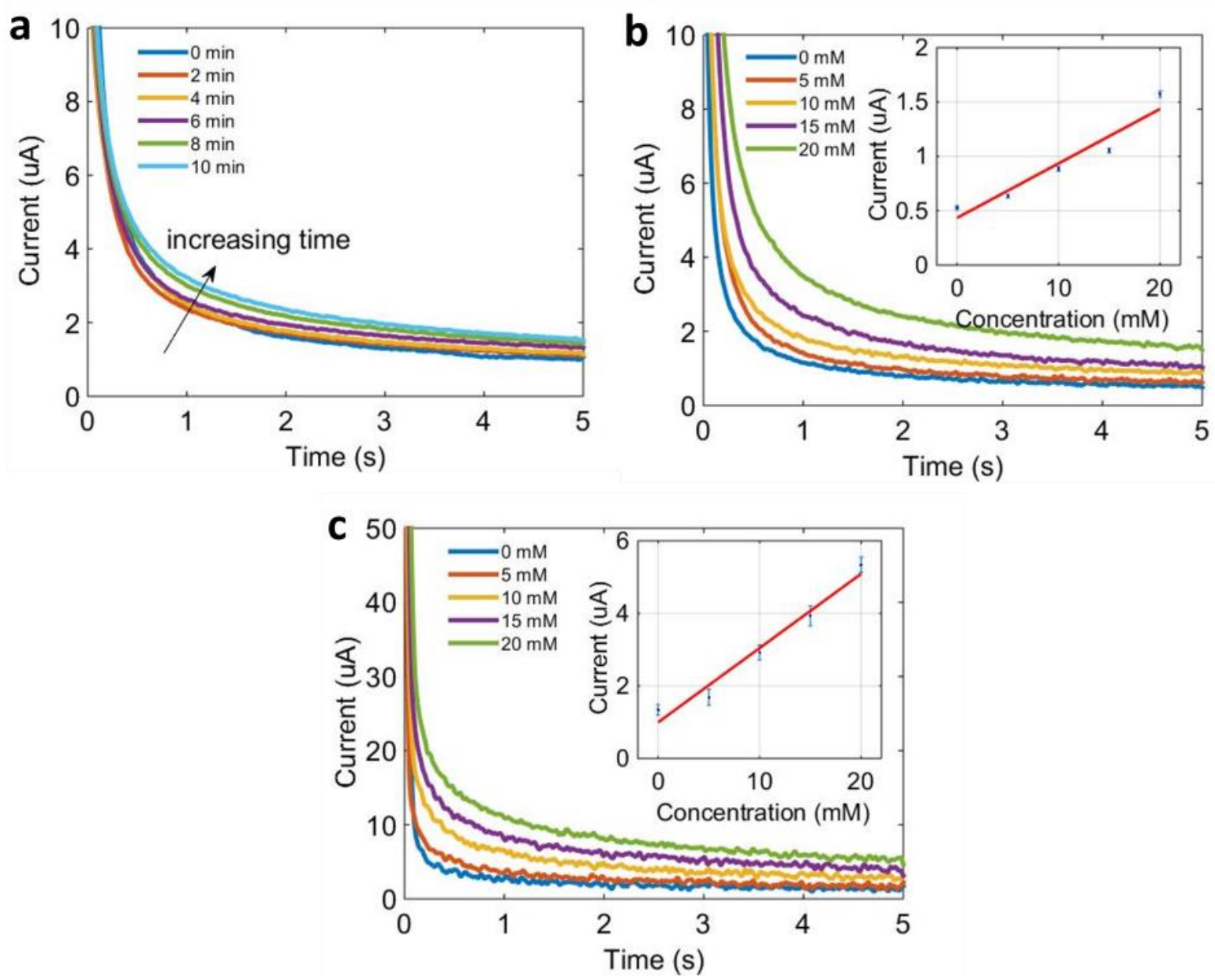

Figure 4. Chronoamperometry results for glucose. a) Effect of reaction time. b) Effect of concentration with the fabricated paper-based devices. c) Effect of concentration with the commercial screen-printed electrodes. The insets in the graphs in b) and c) show the steady-state currents for the range of concentrations and the solid lines represent linear fits to the data with the following equations: $b) y=0.0501 x+0.4339\left(R^{2}=0.926\right)$ and $\left.c\right) y=0.2047 x-0.9966$ $\left(\mathrm{R}^{2}=0.969\right),(\mathrm{n}=5)$.

\section{Conclusion}

In this study, we presented a high-throughput and costeffective method to fabricate paper-based devices for electrochemical assays. In this method, we used a liquid dispenser to deposit carbon paste in a pattern with a $2 \mathrm{D}$ plotter to form the electrodes (working, counter, and reference) required for electrochemical detection of target analytes. This one-step method is both fast and cost-effective: 50 devices can be fabricated in approximately $18 \mathrm{~min}$ and each device costs less than 0.02 USD (based on the cost of materials). A hand-held potentiostat was used to conduct $\mathrm{CV}$ and $\mathrm{CA}$ analyses, which demonstrates the portability of this technique. The devices were validated for $\mathrm{CV}$ and $\mathrm{CA}$ using a model electroactive compound, $\mathrm{K}_{3}\left[\mathrm{Fe}(\mathrm{CN})_{6}\right]$ in $\mathrm{KCl}$. To validate the potential of the presented method for fabricating diagnostic tools, CA analysis was used to conduct a glucose assay. The experimental results showed a linear increase in the current as a function of the glucose concentration.

\section{Acknowledgement}

We acknowledge B. Yenilmez's support on the setup fabrication, and H. Guzel's helps on the experiments.

\section{Author's Contributions}

FG performed the experiments. FG and ST drafted and wrote the manuscript, performed the result analysis.

\section{Ethics}

There are no ethical issues after the publication of this manuscript.

\section{References}

1. Metters, JP, Houssein, SM, Kampouris, DK, et al. 2013. Paperbased electroanalytical sensing platforms. Analytical Methods; 5:103-10.

2. Zhao, C, Thuo, MM, Liu, X. 2013. A microfluidic paper-based electrochemical biosensor array for multiplexed detection of metabolic biomarkers. Science and Technology of Advanced Materials; 14(5):054402. 
3. Amin, R, Ghaderinezhad, F, Li, L, et al. 2017. Continuous-Ink Multiplexed Pen-Plotter Approach for Low-Cost, HighThroughput Fabrication of Paper-Based Microfluidics. Analytical Chemistry; 89 (12): 6351-57.

4. Martinez, AW, Phillips, ST, Whitesides, GM, et al. 2010 Diagnostics for the developing world: microfluidic paper-based analytical devices. Analytical Chemistry; 82 (1): 3-10.

5. Lepowsky, E, Ghaderinezhad, F, Knowlton, S, et al. 2017. Paperbased assays for urine analysis. Biomicrofluidics; 11(5):051501.

6. Li, B, Yu, L, Qi, J, et al. 2017. Controlling Capillary-driven Fluid Transport in Paper-Based Microfluidic Devices Using Movable Valve. Analytical Chemistry; 89 (11): 5707-12.

7. Ghaderinezhad, F, Amin, R, Temirel, M, et al. 2017. Highthroughput rapid-prototyping of low-cost paper-based microfluidics. Scientific Reports; 7:3553.

8. Morbioli, GG, Mazzu-Nascimento, T, Milan, LA, et al. 2017. Improving Sample Distribution Homogeneity in ThreeDimensional Microfluidic Paper-Based Analytical Devices by Rational Device Design. Analytical Chemistry; 89(9):4786-92.

9. Rahbar, M, Nesterenko, PN, Paull, B, and Macka, M. 2017. Geometrical alignment of multiple fabrication steps for rapid prototyping of microfluidic paper-based analytical devices. Analytical Chemistry; 89(22): 11918-11923.

10. Li, Z, Li, F, Hu, J, et al. 2015. Direct writing electrodes using a ball pen for paper-based point-of-care testing. Analyst; 140(16):552635 .

11. Dungchai, W, Chailapakul, O, Henry, CS. 2009. Electrochemical detection for paper-based microfluidics. Analytical chemistry; 81(14):5821-6
12. Yang, Y, Noviana, E, Nguyen, MP, et al. 2016. Paper-based Microfluidic Devices: Emerging Themes and Applications. Analytical chemistry; 89(1):71-91.

13. Shiroma, LY, Santhiago, M, Gobbi, AL, et al. 2012. Separation and electrochemical detection of paracetamol and 4-aminophenol in a paper-based microfluidic device. Analytica Chimica Acta; 725(6):44-50.

14. Liana, DD, Raguse, B, Wieczorek, L, et al. 2013. Sintered gold nanoparticles as an electrode material for paper-based electrochemical sensors. RSC Advances; 3(23):8683-91.

15. $\mathrm{Hu}, \mathrm{C}, \mathrm{Bai}, \mathrm{X}$, Wang, Y, et al. 2012. Inkjet printing of nanoporous gold electrode arrays on cellulose membranes for high-sensitive paper-like electrochemical oxygen sensors using ionic liquid electrolytes. Analytical chemistry; 84(8):3745-50.

16. Ruecha, N, Chailapakul, O, Suzuki, K, et al. 2017. Fully inkjetprinted paper-based potentiometric ion-sensing devices. Analytical Chemistry; 89(19):10608-16.

17. Nie, Z, Deiss, F, Liu, X, et al. 2010. Integration of paper-based microfluidic devices with commercial electrochemical readers. Lab on a Chip; 10(22):3163-9.

18. Dossi, N, Toniolo, R, Pizzariello, A, et al. 2013. Pencil-drawn paper supported electrodes as simple electrochemical detectors for paper-based fluidic devices. Electrophoresis; 34:2085-91.

19. Russo, A, Ahn, BY, Adams, JJ, et al. 2011. Pen-on-paper flexible electronics. Advanced Materials; 23(30):3426-30.

20. Ghosale, A, Shrivas, K, Shankar, R, et al. 2016. Low-Cost Paper Electrode Fabricated by Direct Writing with Silver NanoparticleBased Ink for Detection of Hydrogen Peroxide in Wastewater. Analytical chemistry; 89(1):776-82. 